\title{
A coordinated approach to patient safety for people with adrenal insufficiency
}

\author{
Author: John D Dean ${ }^{\mathrm{A}}$
}

DOI: $10.7861 /$ clinmed.intro.20.4

The publication of 'Guidance for the prevention and emergency management of adult patients with adrenal insufficiency' and how it links to coordinated actions marks a potential landmark in patient safety in the UK. The challenge of reliable emergency treatment for people with known or potential adrenal insufficiency has been known and discussed for many years. The spectrum of patients and scenarios in which effective emergency treatment may be required and the multiple specialties and healthcare sectors involved in coordinating treatment and prevention has landed this in the 'too difficult box' for too long, and thus people have come to harm.

Patient groups and endocrinologists, through the Society for Endocrinology, have been advocating for a coordinated approach. The Royal College of Physicians works with all medical physician specialties, has strong working relationships with the NHS patient safety team and with other healthcare professional representative bodies, and works closely with patients and carers. This has meant that, through the RCP Patient Safety Committee and Joint Professional Medicines Safety Group, we have been able to coordinate work that has resulted in the publication of clear and widely available evidence-based guidelines, the development and launch of a patient-held Steroid Emergency Card, and the underpinning mechanisms for this to be integrated into practice across the NHS through actions outlined in a national Patient Safety Alert.

The Steroid Emergency Card has had an early soft launch in order to make it available for people with known adrenal insufficiency during the COVID 19 pandemic, giving patients and staff more confidence that the right treatment will be given even in the most challenging times, and has had positive patient feedback.

The determination of many for this to be achieved cannot be understated and should be applauded. When professions lead with patients and collaborate within the NHS, much can be achieved.

Author: ${ }^{A}$ clinical director for quality improvement and patient safety, Royal College of Physicians, London, UK 